\title{
Privacy Enhanced Access Control for Outsourced Data Sharing
}

\author{
Mariana Raykova, Hang Zhao, and Steven M. Bellovin \\ Columbia University, Department of Computer Science, \\ New York, NY 10027-7003, USA, \\ \{mariana,zhao, smb\}@cs.columbia.edu
}

\begin{abstract}
Traditional access control models often assume that the entity enforcing access control policies is also the owner of data and resources. This assumption no longer holds when data is outsourced to a third-party storage provider, such as the cloud. Existing access control solutions mainly focus on preserving confidentiality of stored data from unauthorized access and the storage provider. However, in this setting, access control policies as well as users' access patterns also become privacy sensitive information that should be protected from the cloud. We propose a two-level access control scheme that combines coarse-grained access control enforced at the cloud, which provides acceptable communication overhead and at the same time limits the information that the cloud learns from his partial view of the access rules and the access patterns, and fine-grained cryptographic access control enforced at the user's side, which provides the desired expressiveness of the access control policies. Our solution handles both read and write access control.
\end{abstract}

\section{Introduction}

The emerging trend of outsourcing of data storage at third parties - "cloud storage" - has recently attracted tremendous amount of attention from both research and industry communities. Outsourced storage makes shared data and resources much more accessible as users can retrieve them anywhere from personal computers to smart phones. This alleviates data owner from the burden of data management and leaves this task to service providers with dedicated resources and more advanced techniques. By adopting the cloud computing solution, government agencies will drastically save budget and increase productivity by utilizing low-cost and maintenance-free services available on the Internet rather than purchasing, designing and installing new IT infrastructure themselves. Similar benefits could be realized in financial services, health care, education, etc [10].

Security remains the critical issue that concerns potential clients, especially for the banks and government sectors. A major challenge for any comprehensive access control solution for outsourced data is the ability to handle requests for resources according to the specified security policies to achieve confidentiality, and at the same time protect the users' privacy. Several solutions have been proposed in the past $[6,8,12,13,15]$, but none of them considers protecting privacy of the policies and users' access patterns as an essential goal. In this paper we address 
these privacy requirements and propose a mechanism to achieve a flexible level of privacy guarantee for the client. We introduce a two-level access control model that combines fine-grained access control, which supports the precise granularity for access rules, and coarse-grained access control, which allows the storage provider to manage access requests while learning only limited information from its inputs. This is achieved by arranging outsourced resources into units called access blocks and enforcing access control at the cloud only at the granularity of blocks. The fine-grained access control within each access block is enforced at the user's site and remains oblivious to the cloud. The mapping between files and access blocks is transparent to the users in the sense that they can submit file requests without knowing in what blocks the files are contained. While most existing solutions $[2,13,15]$ focus on read request, we present a solution that provides both read and write access control.

\section{$1.1 \quad$ Motivation}

Traditional access control models often make an implicit assumption that the entity enforcing access control policies is also the owner of data. However, in many cases of distributed computing, this assumption no longer holds, and access control policies are enforced at points which should not have direct access to the data content itself, such as data outsourced to an untrusted third party. Hence we need to store data in encrypted form and enforce access control over the encrypted data. The setting of cloud computing falls into this category. The cloud servers are considered to be honest but curious. They will follow our proposed protocol in general, but try to find out as much information as possible based on their inputs. Hence data confidentiality is not the only security concern. Privacy becomes one of the major reasons that drives big companies to build their own private cloud infrastructure rather than making use of the public cloud services.

First of all, access control policies defined by the data owner that govern who can have access to what data become private information with respect to the storage provider. For example, suppose that a business newspaper reports that a secretive company has just hired a new top-level executive who has specialized in a particular field. By watching what other organizations in the company perhaps first research, then development, then procurement and manufacturing - share access groups with this executive, an observer can learn significant details about the company's strategy and progress. This is similar to what military intelligence agencies do when using traffic analysis to determine an enemy's order of battle [7]. In fact, protecting access rules against privacy leakage is a long-standing problem, and has been studied a lot in the past especially for enforcing access control in databases [3]. This problem is mitigated by the use of cryptography as an enforcement mechanism, which translates the access control problem into the question of key management for decryption keys.

A more challenging task, that cannot be solved by data encryption alone, is to protect data access patterns from careful observations on the inputs of the storage provider. Even if data is stored and transferred in an encrypted format, traffic analysis techniques can reveal privacy sensitive information. For example, analysis on the length of encrypted traffic could reveal certain properties of the 
enclosed data; access history could disclose a particular user's access habits and privileges; access to the same data object from multiple users could suggest a common interest or collaborative relationship; a ranking of data popularity can also be built upon access requests that the cloud receives. One trivial solution is to return all encrypted data upon any access request. However, this comes with prohibitive communication costs for data transfer as well as storage and computation costs for decryption at the user's side, which rules out this obvious solution. The question of hiding access pattern is challenging while avoiding work proportional to the total size of all stored files. There have been several cryptographic solutions that realize the notion of oblivious RAM and manage to achieve improved amortized complexity for queries while hiding access patterns $[4,11,1,5]$. However, such solutions are highly interactive and still require communication polylogarithmic in the size of the database, which in the setting of large storage cloud providers, weak client devices and expensive network communication will not be practical (e.g., wireless network communication with limited bandwidth). Furthermore, they assume that the user submitting the query is the owner of all data, which does not fit into our scenario where access control is enforced on data shared by multiple users, not limited to the data owner.

An equally important, but often overlooked, aspect of access control for outsourced data is to enforce the write access. Existing solutions often handle only read requests, which is obviously impractical in a more flexible data sharing scenario. For example, co-workers contribute to the same project document in a collaborative working environment. While data encryption naturally preserves authorization of the read access through key management, the procession of a decryption key implies authorized read access but not necessarily the write. Therefore, different cryptographic schemes are mandatory to manage read and write accesses separately. Further, a full-fledged access control solution should assume no relationship between read and write access rules (a user may have both types of access, only one of them or none).

Therefore we summarize that a privacy-aware access control solution for data sharing in outsourced storage needs to meet the following requirements:

1. it provides data confidentiality by implementing a fine-grained cryptographic access control mechanism;

2. it supports practical and flexible data sharing scheme by handling both read and write operations in the access control model;

3. it enhances data and user privacy by protecting access control rules and access patterns from the storage provider.

\section{Two-level Access Control Model - Solution Overview}

We consider the following scenario: a set of users outsource their data to a remote storage (cloud) provider. These users further would like to be able to share selectively some of their data among themselves. This data sharing should be enabled directly at the cloud through appropriate access control rules that allow users to retrieve all data that they are authorized to access (i.e. not involving the actual data owner). Further, the access control rules governing the data sharing 
and the data that users access are private information of the users and our goal will be to protect this information from the cloud provider.

We distinguish the following three roles in this access control model: the data owner who creates data to be stored at the remote storage in an encrypted format and regulates who has what access to each part of the data; the data user who may have read and write access to the protected data; the cloud provider that stores the encrypted data and responds to access requests. While a solution that enforces access control solely through encryption of the data and appropriate decryption key distribution can achieve complete privacy for the access patterns and access control rules by allowing users to retrieve the whole encrypted database, such an approach will be completely impractical requiring an enormous amount of communication. We suggest a hybrid solution that offers a way to trade off privacy and efficiency guarantees. The basic idea behind it is to provide two levels of access control: coarse-grained and fine-grained. The coarsegrained level access control will be enforced explicitly by the cloud provider and it would also represent the granularity at which he will learn the access pattern of users. Even though the cloud provider will learn the access pattern over all user requests, he will not be able to distinguish requests from different users, which would come in the form of anonymous tokens. The fine-grained access control will be enforced obliviously to the cloud through encryption and would prevent him from differentiating requests that result in the same coarse-grained access control decision but have different fine-grained access pattern.

We realize the above two levels of access control by introducing division of the data resources of the same owner into units called access blocks, which would represent the coarse-level granularity in the system. Now the cloud provider would be able to map user requests to the respective access blocks containing the relevant data only if the user has access to the requested data and without learning which part of the block is accessed. The provider would also not learn the reason for no match: missing data or no access authorization. Our solution does not require users to know the exact access blocks that would contain the data they are searching for. Files might be moved between different blocks, and the only information that users would need in order to request them will be a unique file identifier rather than the id of the current block where the file is residing. We will enable this oblivious mapping of files to blocks using techniques from predicate encryption and some extensions to the scheme [9]. Once a user retrieves the content of the matching block, he would be able to decrypt only the part of the block, which he is authorized to access. We use the ideas of [13] to minimize the decryption keys that need to be distributed for fine-grained access control within access blocks.

While the above suffices for read access control, handling write access control is a little more subtle. The main issue there is that the cloud would need to allow users to submit updates for different parts of an access block without learning which part are updated, and at the same time prevent users authorized to write to one file in the block from writing to another file. In order to facilitate this functionality the cloud provider would accept write updates for blocks only from 
users that provide tokens granting them write access to some part of the block (not revealing which part). These updates will be appended to the content of the block but also the cloud would obliviously tag the updates with the id of the file for which the user has been authorized, but without learning which this file is. We achieve this functionality again through a modification of the searchable ciphertexts in a predicate encryption scheme.

\section{Read Access Control}

In this section, we present in detail the two-level access control scheme for read access only after describing the following techniques applied in our protocol.

\subsection{Techniques}

Fine-Grained Access Control Fine-grained access control is applied to files inside each access block to explicitly enforce access control rules. While the cloud provider is able to determine whether a user submits a legitimate request for some file within a block, he should remain oblivious to the access control rules defined for that file. To guarantee this property the access control view presented to the cloud treats blocks as entities, and the cloud grants a read access by providing the content of an entire block. Fine-grained access control is enforced by encrypting files per block under different keys, and the access control problem is mitigated to appropriate key distribution. Even a user receives the encrypted content of a block, he is able to decrypt only the files that he has access to. Access revocation requires re-encryption of the resource and re-distribution of the new key to the remaining authorized users. Our goal is to minimize the amount of work and interaction between users and the system upon policy updates.

The work of [13] proposes an encryption-based access control solution for outsourced data. Their key distribution is facilitated by the construction of a public tree structure that allows each user to derive file decryption keys using a secret, which he establishes once in the beginning. The leaf nodes in the tree represent initial secrets distributed to users when they join the system, and the internal nodes denote the file decryption keys derivable from leaf nodes using public tokens along a directed path. Any update of the access control rules entails a change in the tree. Access revocation requires re-encryption of affected files.

In our scheme, each user generates a public-private key pair, and the public key is used by data owners as initial secret to construct trees. Hence each user only needs to maintain one key in the system and the distribution of leaf nodes is implicit through the asymmetric key scheme. If some resources are only accessible by a single user, instead of encrypting files using a leaf node (i.e., the public key), we generate a symmetric key for file encryption, which is further encrypted under that public key to avoid expensive computation of asymmetric scheme. In that case, the initial secret needs to be explicitly distributed to an authorized user. Unlike in [13], key derivation tokens have to be protected. First a user's initial secret is generally available to anyone in the system. More importantly, the tree structure itself can reveal certain sensitive information to the cloud. For example, a user having access to one file will have access to all the files along a directed 
path. So the ontent of each node, a pointer to next node and the token to derive next key are all protected under the current encryption key. Thus we can achieve efficient key distribution without requiring any direct interaction between data owners and users beyond some initial set-up assuming only the cloud will be online all the time. A list of algorithms for key distribution and management are summarized in Figure 1.

- Publish $\left(r, o, e_{o}, a c l\right)$ : adds a resource $r$ owned by $o$ with a secret $e_{o}$ and an access control list $a c l=a c l \_r e a d(r)$ for read access.

- Access_Read $(u, r, o)$ : returns the encryption key for a resource $r$ owned by $o$, if $u$ is an authorized user.

- Find_Chain $(u, r)$ : finds the shortest chain of tokens from the secret key of user $u$ to derive the decryption key for resource $r$.

- Compute_Key $(u$, chain $)$ : derives the secret key for a user $u$ given a chain of transition tokens.

- Find_Resources $(u, r)$ : finds the set of nodes that lie on any path from the user $u$ to the node corresponding to resource $r$.

- Update $(r, a c l)$ : if there is another resource with the same access control list $a c l$, i.e., there is a node in the tree accessable exactly by a subset of users in $a c l$, then encrypt $r$ with the key contained in that node. Otherwise, encrypt $r$ with a new key, add a new node containing this key to the tree and add appropriate edges to connect the new node to the users who have access to $r$. (Note that certain subgroups of the users in acl might already have a shared key through another node in tree, and in that case we connect to that node rather than all the users' nodes separately.)

Fig. 1: Algorithms for key distribution and management for fine-grained AC.

Coarse-Grained Access Control The main goal to achieve at the level of coarse-grained access control is to enable the cloud provider to obliviously match a user's request to an access block without learning which part of the block the user is authorized to access. In addition we provide unlinkability among multiple requests for the same resource even if coming from the same user, which further protects users' access patterns from the cloud provider. In order to achieve these goals we apply the predicate encryption scheme of [9]. Observing that in this scheme ciphertext can be re-randomized even without knowledge of the secret key, we define a re-randomization algorithm in Definition 1.

Definition 1. A re-randomizable predicate encryption scheme consists of the following algorithms:

- $\operatorname{Setup}\left(1^{n}\right)$ : produces a master secret key $S K$ and public parameters;

- $\operatorname{Enc}_{S K}(x)$ : encrypts an attribute $x$ using key $S K$;

- $\operatorname{GenKey}_{S K}(f)$ : generate a decryption key $S K_{f}$ associated with a function $f$;

- $\operatorname{Dec}_{S K_{f}}(c)$ : outputs 1 if the attribute encrypted in $c=E_{n c_{S K}}(x)$ satisfies $f$, i.e. $f(x)=1$, and output a random value, otherwise;

- Rand $(c)$ : computes a new encryption $c^{\prime}$ of the value encrypted in $c$ but with different randomness without the secret key.

We present the predicate encryption scheme of [9] and the instantiation of the function $\operatorname{Rand}(c)$ for that scheme in Appendix A. This scheme handles a class of 
functions $f$, which includes polynomials of bounded degree. We use polynomial functions of the type $f(x)=\left(x-i d_{1}\right) \cdots\left(x-i d_{n}\right)$, to implement coarse-grained access control. Figure 2 present a list of algorithms to enforce access control on the block level granularity without revealing the exact files that are being accessed insider a block. The algorithm File_Access_Check grants access if the submitted access token matches any of the files in the block without revealing the file identity. The request token produced by File_Access_Request is an encryption that does not leak information about the file id it contains.

- Block_Access_Setup: data owner runs $\operatorname{Setup}\left(1^{n}\right)$, publishes the public parameters and keeps the master secret key $S K$. For files $i d_{1}, \ldots, i d_{n}$ in each block, he computes $S K_{f}=\operatorname{GenKey}_{S K}(f)$ for $f(x)=\left(x-i d_{1}\right) \cdots(x-$ $i d_{n}$ ) and sends $S K_{f}$ to the cloud provider.

- File_Access_Authorization: data owner provides access to a file $i d$ by sending $c_{i d}=\operatorname{Enc}_{S K}(i d)$ to an authorized user.

- File_Access_Request: user generates a token $t_{i d}=\operatorname{Rand}\left(c_{i d}\right)$ for file $i d$.

- File_Access_Check: upon receiving a request token $t$, the cloud computes $\operatorname{Dec}_{S K_{f}}(t)$ for each block, and returns those blocks that compute to 1 .

Fig. 2: Algorithms for enforcing coarse-grained $\mathrm{AC}$ at the access block level.

\subsection{Read Access Control}

We present a read access control solution consisting of the following algorithms. Unless explicitly stated, all the actions are performed by individual data owners.

- System Setup: At the fine-grained level, files are distributed into access blocks. Generate a tree graph per block by running $\mathbf{P u b l i s h}\left(r, o, e_{o}, a c l\right)$ for each resource $r$ owned by $o$ with initial ACLs, and encrypt resources using keys from the tree graph. At the coarse-grained level, each data owner computes parameters for a predicate encryption scheme. Then he constructs a separate tree graph over all resources he owns to distribute authorization tokens of the form $c_{i d}=\operatorname{Enc}_{P K}(i d)$ (i.e., now tree nodes contain authorizations tokens rather than file decryption keys). Finally, data owner computes a key $S K_{f}=\operatorname{GenKey}_{S K}(f)$ per block where $f$ is the polynomial derived from the ids of the files contained in that block as described above, and gives this key to the cloud provider, which will use it to obliviously check read access on authorization tokens.

- Access Authorization: At the fine-grained level, add a leaf node containing the new user's public key to the corresponding tree graph with encryption keys. Update the graph by adding new internal nodes and appropriate edges if necessary. Update file encryptions if new internal nodes were added previously. At the coarse-grained level, perform similar operations with respect to the tree graph containing read access tokens.

- Access Request: At the fine-grained level, an authorized user $u$ derives the decryption key from the tree graph for resource $r$ by calling Find_Chain $(u, r)$, Find_Resources $(u, r)$ and Compute_Key $(u$, chain $)$. At the coarse-grained level, he calls the same set of functions but to query the tree graph with access tokens and find token $c_{i d}=\operatorname{Enc}_{S K}(i d)$ for the requested file $i d$, and then submit a randomized token $t_{i d}=\operatorname{Rand}\left(c_{i d}\right)$ to the cloud. 
- Access Check: At the fine-grained level, only authorized users can derive the correct decryption key for any file using the public tree structure. At the coarsegrained level, the cloud provider executes File_Access_Check to identify the block that contains the requested file.

- Access Rule Update: At the fine-grained level, changes are applied immediately upon policy updates. If the policy update involves access revocation, the data owner changes the encryption of corresponding files. The data owner identifies the blocks affected by those files and updates their tree graphs with decryption keys. The changes at the coarse-grained level happen at longer intervals of time, the length of which would depend on the resources of the data owner. They involve updating of the tree graph with access tokens.

\section{Write Access Control}

Enforcing write access control presents more challenges, mainly for the fact that access control through data encryption does not apply to cases when data can be modified. Without revealing fine-grained access control policies, it is not guaranteed that a user will modify only files that he is granted write access to. An unauthorized user can overwrite and destroy data without being detected by the cloud, regardless of whether he has the read privilege. A trivial solution is to rely on the cloud provider to restrict the memory regions to which users may submit changes, which however reveals to the cloud access rules and access patterns.

The approach that we adopt is to record modifications of files in new regions of memory without overwriting previous content. The coarse-grained access control enforced by the cloud allows users to submit changes for files only if they can demonstrate write permission for some resource in that block, without revealing the exact content to be changed. At the fine-grained level, a public encryption scheme is used to separate read and write privileges by providing a key pair for each file. The only information that the cloud tags to each file change obliviously contains implicit information on file identifier. However, using a submitted write authorization token directly as an update identifier will enable users with only read access to copy and reuse it later to obtain write privilege. To prevent this undesired situation, we take advantage of the predicate encryption ciphertexts constituting access tokens, which allows us to use part of the token as identifier. We generate predicate that allows users with read access to identify relevant updates, but this identifier on its own is not sufficient to grant write access.

\subsection{Techniques}

File Encryption We apply an asymmetric encryption scheme to handle all possible combinations of read and write access to a file. Since such scheme is computationally expensive for large size of data, file content is still encrypted using a symmetric key (e.g., AES), which is further encrypted under the public key. Two trees are constructed for key distribution per block - one for the public (encryption) keys and the other for the private (decryption) keys. These two trees share the same set of internal nodes for an one to one correspondence between public and private key pair. Only files readable and writable by the same set of users can share the same public key pair. 
Access Authorization Tokens Two trees are constructed by each data owner for the distribution of read and write access tokens respectively.

File Identifiers for Write Updates We observe that the write authorization token is a valid encryption for a predicate encryption that provides polynomials evaluation, and the structure of the encrypted plaintext for access to file $i d$ is a vector of the form $\left(1, i d, i d^{2}, \ldots, i d^{n}\right)$, where $n$ is the number of files placed in a block. The structure of the ciphertext allows it to be split into parts where one part is an encryption of the vector $\left(1, i d, i d^{2}, \ldots, i d^{k}\right)(k<n, n>2)$, which is no longer a valid write access token for that file, but can still be used identify file updates for users with read privilege. This can be achieved using a decryption predicate for a polynomial of degree $k$ that has $i d$ as a zero point. (See Appendix A for details.)

\subsection{Integrated Read and Write Access Control}

We realize the above proposal for the write access control enforcement and describe an integrated solution for both read and write access. Because of space constraints we describe only the functionality associated with write access enforcement. The read access is the same as the construction in the previous section with the exception that once a client has retrieved a block he needs to identify both the original encryption of the file as well as all updates for that file. The latter will be achieved using an additional key (a new part in his authorization token) that will allow him to identify the valid updates submitted for that file.

- Setup: At the fine-grained level, construct a key distribution tree per block based on read access rules. For each node in the tree, generate a public-private key pair $\left(s k_{n}, p k_{n}\right)$, but only store the secret key $s k_{n}$. Construct another tree with the same set of nodes to store the public key $p k_{n}$, with edges determined by write access rule. For each file $i d$ generate a AES key $s k_{i d}^{a e s}$ for encryption, and append to the ciphertext $\operatorname{Enc}_{p k_{n}}\left(s k_{i d}^{a e s}\right)$. At the coarse-grained level, each data owner generates two sets of parameters $\left(p k^{\prime}, s k^{\prime}\right)$ and $\left(p k ", s k^{\prime \prime}\right)$ for the predicate encryption. Then he constructs a tree graph, where each node contains read access token $\operatorname{Enc}_{p k_{r a}^{\prime}}(i d)$ (used by the cloud provider to check the read access) and $S K_{x-i d}=$ GenKey $_{s k_{r a}^{\prime \prime}}(f)$ where $f(x)=x-i d$ (used by the user to identify all updates to the file within the retrieved block). Similarly, construct another tree to distribute write access tokens $\operatorname{Enc}_{p k_{w a}}(i d)$.

- Access Authorization: At the coarse-grained level, extend the trees with read and write access tokens with new leaves for the new user and update the edges according to his read and write permissions. This may involve splitting of nodes and re-encrypting files with new keys if the user has read access only to a subset of files that have been encrypted with the same key.

- Write Access Request: At the fine-grained level, obtain the encryption key $p k_{n}$ for the file to be updated from the write tree. Encrypt the new content for that file with key $p k_{n}$. At the coarse-grained level, submit to the cloud a re-randomized copy of the write authorization token for that file.

- Write Access Check: At the fine-grained level, a user can modify a file only if he has the encryption key and the write authorization token. Upon read he will check at the end of a block a list of updates with valid write access 
tokens. At the coarse-grained level, the cloud finds if there is a block for which the authorization token grants write access. The write access token is of the form $\left(C_{0},\left\{C_{1, i}, C_{2, i}\right\}_{i=1}^{n}\right)$, and the cloud uses the first components $\left(C_{0},\left\{C_{1, i}, C_{2, i}\right\}_{i=1}^{2}\right)$ as an identifier for updates appended to a block.

- Write Access Rule Update: Update per-block trees for encryption keys and the tree for distributing write access tokens accordingly.

\subsection{An Example}

To facilitate our discussion, consider a system with five users $U=\{A, B, C, D$, $E$ \}. Let $R_{o}$ denote the set of resources owner by user $o \in U$, and we have $R_{A}=\left\{r_{1}, r_{2}, r_{3}, r_{4}\right\}, R_{B}=\left\{r_{5}, r_{6}, r_{7}\right\}$ and $R_{C}=R_{D}=R_{E}=\emptyset$. Access control lists (ACLs) are used to represent fine-grained level access policies, and the owner of each resource automatically entails both read and write access privilege. At the coarse-grained level, user $A$ maintains two blocks $b_{1}=\left\{r_{1}, r_{2}\right\}$ and $b_{2}=\left\{r_{3}, r_{4}\right\}$, and user $B$ maintains a single block $b_{3}=\left\{r_{5}, r_{6}, r_{7}\right\}$.

1. acl_read $\left(r_{1}\right)=\{A, B, C\}$, acl_write $\left(r_{1}\right)=\{A, B, C\}$;

2. acl_read $\left(r_{2}\right)=\{A, B, C\}$, acl_write $\left(r_{2}\right)=\{A, B, C\}$;

3. acl_read $\left(r_{3}\right)=\{A, E\}$, acl_write $\left(r_{3}\right)=\{A\}$;

4. acl_read $\left(r_{4}\right)=\{A, B, C, E\}$, acl_write $\left(r_{4}\right)=\{A, D\}$;

5. acl_read $\left(r_{5}\right)=\{A, B\}$, acl_write $\left(r_{5}\right)=\{A, B, C\}$;

6. acl_read $\left(r_{6}\right)=\{B, C, D\}$, acl_write $\left(r_{6}\right)=\{B, D, E\}$;

7. acl_read $\left(r_{7}\right)=\{A, B, C, D, E\}$, acl_write $\left(r_{7}\right)=\{A, B, C, D, E\}$.

Follow the above example, two trees are constructed per block for read and write access respectively at the fine-grained level in Figure 3. Each block stores files owned by a single user (shaded), and a public key pair is generated for each contained resource $r_{i}$, where $s k_{i d}$ is stored in the read tree and $p k_{i d}$ in the write tree. Leaf nodes $v_{1 n}^{* j}$ store users' initial public keys, which are connected to internal nodes following key derivation paths (think links). Each row in the table states $r_{i}$ in block $b_{j}$ is associated with key $s k_{i d}\left(p k_{i d}\right)$ at vertex $v_{m n}^{R j}\left(v_{m n}^{W j}\right)$. Different ACLs on read and write for the same resource entail different labels of user list, e.g., $v_{31}^{R 2}$ is labeled as $[A B C E]$ since $\operatorname{acl} \_r e a d(r 4)=\{A, B, C, E\}$; whereas $v_{31}^{W 2}$ is labeled as $[A D]$ given acl_write $(r 4)=\{A, D\}$. In Figure 3(a), both trees share the same set of vertexes and edges, as acl_read $\left(r_{1}\right)=\operatorname{acl} \_r e a d\left(r_{2}\right)=$ acl_write $\left(r_{1}\right)=$ acl_write $\left(r_{2}\right)=\{A, B, C\}$. Figure 4 depicts tree graphs per data owner to distribute read and write access tokens respectively at the coarsegrained level. (Due to space limitation, coarse-grained graphs for resources owned by user $B$ are omitted.) Each row in the table states $r_{i}$ is associated with an unique read (write) access token $E n c_{p k^{\prime}{ }_{r a}}(i d)\left(E n c_{p k_{w a}}(i d)\right)$ encrypted on its $i d$ and stored at vertex $v_{m n}^{R O}\left(v_{m n}^{W O}\right)$. For example, $r_{1}$ and $r_{2}$ are now given different access tokens at vertexes $v_{21}^{* A}$ and $v_{22}^{* A}$ respectively. Each authorized write operation requires an additional update token $S K_{x-i d}$ distributed to authorized users the same way as read access tokens.

\section{Analysis}

\subsection{Security Guarantees}

Our two-leveled access control scheme provides the following privacy guarantees for data owners and users in the system: 


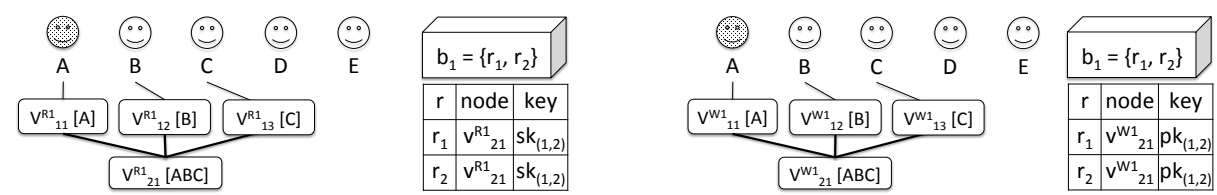

(a) Encryption policy graph of read-and-write access for block $b_{1}$

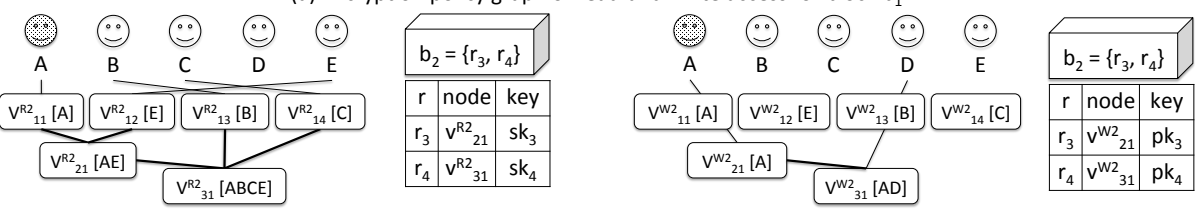

(b) Encryption policy graph of read-and-write access for block $b_{2}$

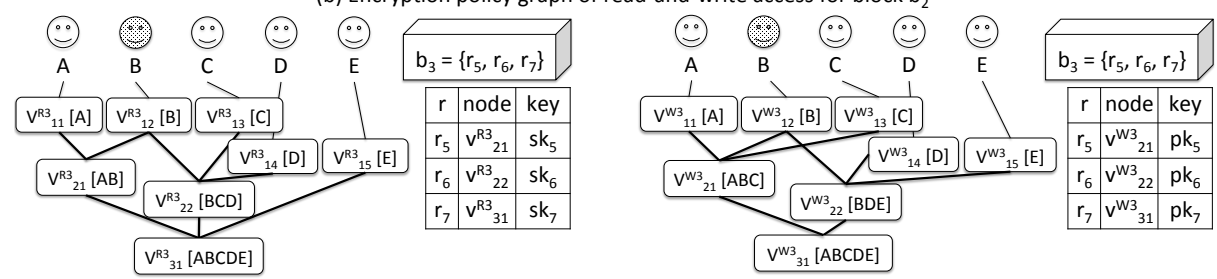

(c) Encryption policy graph of read-and-write access for block $b_{3}$

Fig. 3: Tree graphs per block for read and write access at the fine-grained level.

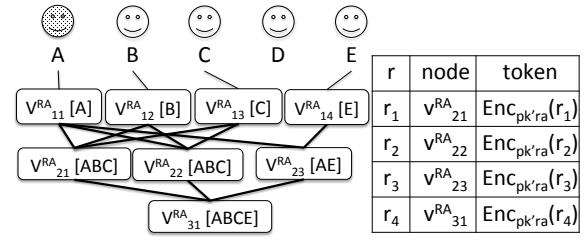

(a) Read access token graph for owner $A$

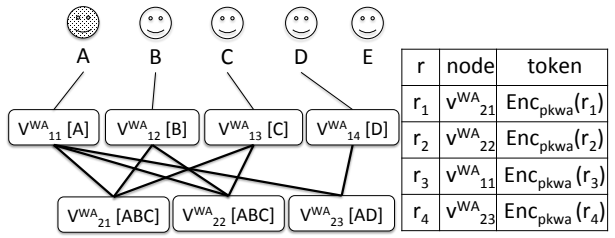

(b) Write access token graph for owner $A$

Fig. 4: Distribution of read and write access tokens at the coarse-grained level.

Read Access For the privacy of the data owners, the cloud provider does not learn any of the content of the files that he stores. The cloud learns the frequency of access to particular blocks but not the exact files that have been accessed within a block. For users' privacy, the cloud provider cannot relate access requests to particular users', neither can he infer which requests were submitted from the same user. However, he can observe the block access pattern from the requests of all users. The data owner does not learn anything about the access requests for the data.

Write Access For privacy of the data owners, the cloud provider learns how often update requests are submitted for each block but without finding out which files have been written. Similarly to the read requests, write requests coming from the users are anonymous and unlinkable. Thus the cloud provider cannot learn anything about the access behavior of a particular user, but only a cumulative view over the requests from all users.

\subsection{Performance Analysis}

Read Access During setup, data owners compute of the authorization trees with decryption keys and access tokens. The work is proportional to the number of files in the database and the number of users. In order to authorize or to 
revoke file access to a user, data owner updates the tree with decryption keys and the tree with access tokens: in the worst case proportional to the depth of the trees. The updates for the tree with the access tokens can be executed at larger intervals of time to achieve better amortized efficiency for updates. For users, retrieving access tokens requires reading the coarse-level tree with access tokens for the data of a particular provider. Decryption keys retrieval can be proportional to the number of files that the user is authorized to access. For the cloud provider, in order to map an access request to a particular block the cloud provider will have to execute the File_Access_Check function for the submitted token and each block. We describe in the following optimization section how we can reduce the costs estimated here.

Write Access From the perspective of a data owner, the enforcement of write access control requires duplication of the tree structures that were necessary for the read access control but this time with credentials necessary for the write access. This comes as an overhead in the setup phase when these structures are computed by the data owner and also each update of the access rules will necessitate update of both types of trees since the encryption and decryption (relevant for write and read access) need to be synchronized. Also periodically the data owner would need to process the blocks and compact the updates for each file back in its initial memory location. For a user, the size of the blocks that he receives, and hence the time he needs to locate the file and its updates at read access, will increase depending on the frequency of the updates for a block as well as the time period at which the data owner processes the blocks and brings the updates back in place. The cloud provider would need to transfer larger blocks including both the original files as well as the updates. He would need to compute the identification tag for each authorized write update, which requires constant time.

Optimizations. Some optimizations that help improve the performance of the scheme are as follows. If the user has enough memory, he can cache both authorization tokens and decryption keys for multiple accesses of the same files. This optimization applies to the read and the write access tokens as well as the decryption key for read. The only exception is the encryption key for write access - the user should always derive the current public encryption key for the file, which he wants to update since if the key has been changed, he will not be able to detect it and will submit an invalid update. Similarly the user can cache the identifier of the block in which a file is located and use it in repeated requests, which will save the search time at the cloud avoiding checks of all blocks. Further the user can trade-off the privacy guarantee for his request within its block for smaller communication overhead by revealing the exact memory address of the file after proving that he is authorized to access the block.

\subsection{Discussion}

Choosing the granularity for the access blocks in the read and write access control schemes affects the privacy guarantees for the scheme as well as its efficiency performance. The right granularity for each specific usage scenario will depend on the privacy and efficiency requirements for it, the expected patterns of access 
to the files and the expected frequency of access control rules' updates. The following points should be taken into consideration when choosing how to divide the files into access blocks: the size of a block should depend on the expected bandwidth of the clients and the acceptable delays for the system. Files that contain "complementary" information, i.e., a user is likely to access only one of a these files (e.g. a file to sell stocks, a file to buy stocks) should be located in the same block since their access pattern is highly sensitive. Data that requires frequent updates should be split into smaller blocks since the size of those blocks will grow faster. Accessing files with frequently changing access rules will require derivation of the corresponding decryption keys, which is proportional to the number of files in the block, such files should be located in blocks with fewer items (that can still be of big size). Since the view of the cloud provider of the access requests amounts to the frequency at which each access block is matched, files that are expected to have high access rates should be distributed across different blocks.

\section{Related Work}

Existing access control solution in outsourced storage usually apply cryptographic methods by disclosing data decryption keys only to authorized users. [8] proposed a cryptographic storage system, called Plutus, which arranges files with similar attributes into filegroups, applying two-level file encryption and distinguishes read and write access. [6] designed a secure file system to be layered over insecure network and P2P file system, like NFS. Each file is attached a meta data containing the file's access control list. [15] defines and enforces fine-grained access control policies based on data attributes, and delegates most of computation tasks to untrusted cloud server without disclosing data content. [12] proposed a cloud storage system, called CloudProof, that enables meaningful security Service Level Agreements (SLAs) by providing a solution to detect violations of security properties, namely confidentiality, integrity, write-serializability, and read freshness. The problem presented in this paper shares some similarity with the proposals in $[14,2]$. [14] introduced a practical oblivious data access protocol using pyramid-shaped database layout and an enhanced reordering techniques to ensure access pattern confidentiality. [2] proposed a shuffle index structure, adapting traditional B-tree, to achieve content, access and pattern confidentiality in the scenario of outsourced data. All those proposals focus on one or more aspects, such as scalability, efficiency, minimizing key distribution, etc., but none of them consider privacy issues as well as write access control.

\section{Conclusion}

We presented a two-level access control scheme enabling data sharing in outsourced storage, like the cloud environment. The fine-grained and the coarsegrained access control schemes complement each other to achieve both data confidentiality and privacy protection on access patterns. To the best of our knowledge, we are the first to handle both read and write access rights entailing a more practical data sharing solution. As follow-on work, we will conduct experiments on a full implementation of our scheme. As a more ambitious goal, we 
would like to further extend our scheme for a complete solution that guarantees both security and privacy protection for a remote file storage system.

\section{References}

1. Ivan Damgård, Sigurd Meldgaard, and Jesper Buus Nielsen. Perfectly secure oblivious ram without random oracles. In TCC, 2011.

2. S. De Capitani di Vimercati, S. Foresti, S. Paraboschi, G. Pelosi, and P. Samarati. Efficient and private access to outsourced data. In Proc. of the 31st International Conference on Distributed Computing Systems (ICDCS 2011), Minneapolis, Minnesota, USA, June 2011.

3. S. De Capitani di Vimercati, S. Foresti, and P. Samarati. Recent advances in access control. In M. Gertz and S. Jajodia, editors, Handbook of Database Security: Applications and Trends. Springer-Verlag, 2008.

4. O. Goldreich and R. Ostrovsky. Software protection and simulation on oblivious RAMs. Journal of the ACM (JACM), 43(3):473, 1996.

5. Michael T. Goodrich and Michael Mitzenmacher. Privacy-preserving access of outsourced data via oblivious ram simulation. In in Proc. International Colloquium on Automata, Languages and Programming, ICALP'11, 2011.

6. Eu jin Goh, Hovav Shacham, Nagendra Modadugu, and Dan Boneh. Sirius: Securing remote untrusted storage. In in Proc. Network and Distributed Systems Security (NDSS) Symposium 2003, pages 131-145, 2003.

7. David Kahn. The Codebreakers. Macmillan, New York, 1967.

8. Mahesh Kallahalla, Erik Riedel, Ram Swaminathan, Qian Wang, and Kevin Fu. Plutus: Scalable secure file sharing on untrusted storage. In USENIX Conference on File and Storage Technologies, 2003.

9. Jonathan Katz, Amit Sahai, and Brent Waters. Predicate encryption supporting disjunctions, polynomial equations, and inner products. In Proceedings of the theory and applications of cryptographic techniques 27th annual international conference on Advances in cryptology, EUROCRYPT'08, pages 146-162, Berlin, Heidelberg, 2008. Springer-Verlag.

10. Vivek Kundra. Tight budget? look to the 'Cloud'. The New York Times, 2011. http://www.nytimes.com/2011/08/31/opinion/ tight-budget-look-to-the-cloud.html?_r=1.

11. B. Pinkas and T. Reinman. Oblivious RAM Revisited. Advances in CryptologyCRYPTO 2010, pages 502-519, 2010.

12. Raluca Ada Popa, Jacob R. Lorch, David Molnar, Helen J. Wang, and Li Zhuang. Enabling security in cloud storage slas with cloudproof. In in Proc. USENIX Annual Technical Conference ATC'11, 2011.

13. S. De Capitani di Vimercati, S. Foresti, S. Jajodia, S. Paraboschi, G. Pelosi, and P. Samarati. Encryption-based policy enforcement for cloud storage. In Proceedings of the 2010 IEEE 30th International Conference on Distributed Computing Systems Workshops, ICDCSW '10, pages 42-51, 2010.

14. Peter Williams, Radu Sion, and Bogdan Carbunar. Building castles out of mud: practical access pattern privacy and correctness on untrusted storage. In Proceedings of the 15th ACM conference on Computer and communications security, CCS '08, pages 139-148, New York, NY, USA, 2008. ACM.

15. Shucheng Yu, Cong Wang, Kui Ren, and Wenjing Lou. Achieving secure, scalable, and fine-grained data access control in cloud computing. In Proceedings of the 29th conference on Information communications, INFOCOM'10, pages 534-542, Piscataway, NJ, USA, 2010. IEEE Press. 


\section{A Predicate Encryption and Extensions}

We present the construction of predicate encryption of [9] as follows:

$-\operatorname{Setup}\left(1^{n}\right)$ : Choose primes $p, q$ and $r$ and groups $\mathbf{G}_{p}, \mathbf{G}_{q}$ and $\mathbf{G}_{r}$ with generator $g_{p}, g_{q}$ and $g_{r}$ respectively. Let $\mathbf{G}=\mathbf{G}_{p} \times \mathbf{G}_{q} \times \mathbf{G}_{r}$. Choose $R_{1, i}, R_{2, i} \in \mathbf{G}_{r}$, $h_{1, i}, h_{2, i} \in \mathbf{G}_{p}$ uniformly at random for $1 \leq i \leq n$ and $R_{0} \in \mathbf{G}_{r}$. The public parameters for the scheme are $\left(N=p q r, \mathbf{G}, \mathbf{G}_{T}, e\right)$. The public key $P K$ and master secret key $S K$ are defined as follows:

$$
\begin{gathered}
P K=\left(g_{p}, g_{r}, Q=g_{q} \cdot R_{0},\left\{H_{1, i}=h_{1, i} \cdot R_{1, i}, H_{2, i}=h_{2, i} \cdot R_{2, i}\right\}_{i=1}^{n}\right), \\
S K=\left(p, q, r, g_{q},\left\{h_{1, i}, h_{2, i}\right\}_{i=1}^{n}\right) .
\end{gathered}
$$

- $\operatorname{Enc}_{S K}\left(x_{1}, \ldots, x_{n}\right):$ Choose randoms $s, \alpha, \beta \in \mathbf{Z}_{N}, R_{3, i}, R_{4, i} \in \mathbf{G}_{r}$ for $1 \leq$ $i \leq n$, then output the following ciphertext:

$$
C=\left(C_{0}=g_{p}^{s},\left\{C_{1, i}=H_{1, i}^{s} \cdot Q^{\alpha \cdot x_{i}} \cdot R_{3, i}, C_{2, i}=H_{2, i}^{s} \cdot Q^{\beta \cdot x_{i}} \cdot R_{4, i}\right\}_{i=1}^{n}\right) .
$$

- $\operatorname{GenKey}_{S K}\left(v_{1}, \ldots, v_{n}\right)$ : Choose randoms $r_{1, i}, r_{2, i} \in \mathbf{Z}_{p}$ for $1 \leq i \leq n, R_{5} \in$ $\mathbf{G}_{r}, f_{1}, f_{2} \in \mathbf{Z}_{q}$ and $Q_{6} \in \mathbf{G}_{q}$, then output $S K_{\mathbf{v}}$ that consists of

$$
\left(K=R_{5} \cdot Q_{6} \cdot \prod_{i=1}^{n} h_{1, i}^{-r_{1, i}} \cdot h_{2, i}^{-r_{2, i}},\left\{K_{1, i}=g_{p}^{r_{1, i}} \cdot g_{q}^{f_{1} \cdot v_{i}}, K_{2, i}=g_{p}^{r_{2, i}} \cdot g_{q}^{f_{2} \cdot v_{i}}\right\}_{i=1}^{n}\right)
$$

$-\operatorname{Dec}_{S K_{f}}(c)$ : The decryption algorithm outputs 1 if and only if

$$
e\left(C_{0}, K\right) \prod_{i=1}^{n} e\left(C_{1, i}, K_{1, i}\right) \cdot e\left(C_{2, i}, K_{2, i}\right)=1
$$

We define an algorithm called $\operatorname{Rand}(C)$ that re-randomizes any ciphertext produced by the predicate encryption. Given a ciphertext of form $\left(C_{0},\left\{C_{1, i}, C_{2, i}\right\}_{i=1}^{n}\right)$, choose a random $s^{\prime} \in \mathbf{Z}_{N}$ and output $C^{\prime}=C_{0} \cdot g_{p}^{s^{\prime}},\left\{C_{1, i} \cdot H_{1, i}^{s^{\prime}}, C_{2, i} \cdot H_{2, i}^{s^{\prime}}\right\}_{i=1}^{n}$. The resulting ciphertext is the same as freshly generated ciphertext for the encrypted value using random value $s+s^{\prime}$, if $s$ was the value used in $C$.

Now we look closely at the instantiation of the predicate encryption scheme that handles polynomial evaluation as its predicate. In this case the predicate $\left(v_{1}, \ldots, v_{n}\right)$ consists of the coefficients of the polynomial that is being evaluated and the attribute vector that is used for an evaluation point $x$ is of the form $\left(1, x, x^{2}, \ldots, x^{n-1}\right)$. The ciphertext for the encryption of $\left(1, x, x^{2}, \ldots, x^{n-1}\right)$ has components $\left(C_{0},\left\{C_{1, i}, C_{2, i}\right\}_{i=1}^{n}\right)$, where $C_{1, i}, C_{2, i}$ correspond to the vector point $x^{i-1}$. Thus we can view the first view components of the ciphertext $\left(C_{0},\left\{C_{1, i}, C_{2, i}\right\}_{i=1}^{2}\right)$ as an encryption of the vector $(1, x)$ that can be used for evaluation of predicates that are linear functions.

We use the above observation in the instantiation of the tags that the cloud derives for each of the accepted write updates. He uses the token that the client has used to prove his write access to a particular block, which a predicate encryption ciphertext $\left(C_{0},\left\{C_{1, i}, C_{2, i}\right\}_{i=1}^{n}\right)$, to derive identifier for the files with which the submitted update will be associated by taking the first part of the ciphertext $\left(C_{0},\left\{C_{1, i}, C_{2, i}\right\}_{i=1}^{2}\right)$. This identifier cannot be used as a write access token since it is missing substantial part of the ciphertext, and no party without the master secret key can extend an identifier to a valid write token. Also any party that has read access to the file associated with the update will be given a key that would allow it to recognize the updates for that file. This key is the predicate corresponding to the linear function that evaluates to zero at the file id. 